\title{
Tratamento de hemangiomas da região de cabeça e pescoço: revisão integrativa da
}

\section{literatura}

\author{
Treatment of hemangiomas in the head and neck region: integrative literature review \\ Tratamiento de hemangiomas en la región de cabeza y cuello: revisión integrativa de la literatura
}

Recebido: 2912/2021 | Revisado: 03/01/2022 | Aceito: 07/01/2022 | Publicado: 11/01/2022

Débora Belém Sampaio

ORCID: https://orcid.org/0000-0003-3995-0137

Faculdade Santa Maria, Brasil

E-mail: dbs-debora@hotmail.com

Wliana Pontes de Lima

ORCID: https://orcid.org/0000-0002-4384-580X Universidade Estadual da Paraíba, Brasil

E-mail: wli_pontes@outlook.com

Ingrid de Sousa Matias

ORCID: https://orcid.org/0000-0003-4857-3206

Faculdade Santa Maria, Brasil

E-mail: ingridmatias321@gmail.com

Isabela Santos Saraiva

ORCID: https://orcid.org/0000-0002-5809-6776

Faculdade Santa Maria, Brasil

E-mail: isabella_noelle96@hotmail.com

Matheus Leite Ferreira

ORCID: https://orcid.org/0000-0002-8303-1851

Faculdade Santa Maria, Brasil

E-mail: mleite272@gmail.com

Vitória Maranhão Ramos

ORCID: https://orcid.org/0000-0003-0255-0455 Faculdade Santa Maria, Brasi

E-mail: vitoria_viva2010@hotmail.com

Pedro José Targino Ribeiro

ORCID: https://orcid.org/0000-0002-0671-9361 Faculdade Santa Maria, Brasil

E-mail: profpedrotargino@gmail.com

\section{Resumo}

Este estudo objetivou identificar as principais formas de tratamento dos hemangiomas de cabeça e pescoço. Trata-se de uma revisão integrativa da literatura, que teve como questão norteadora a abordagem terapêutica dos hemangiomas. Foi realizado um levantamento bibliográfico nas bases de dados Scielo e Pubmed, entre os anos de 2009 a 2019. A busca resultou na seleção de 28 artigos, após realizou-se averiguação dos seguintes critérios de elegibilidade: acesso livre, publicações nos idiomas português, inglês ou espanhol e estudos cujos títulos e resumos mostraram-se em consonância com o propósito desta revisão. Ao final, selecionaram-se 5 artigos. Foram excluídas, a literatura cinzenta e duplicatas. Mediante a análise crítica dos estudos, estimou-se que apenas 10 a $20 \%$ dos hemangiomas necessitam de tratamento. As opções terapêuticas para o hemangioma são diversas, variando conforme as características dos pacientes e peculiaridades da lesão, como localização, tamanho, estágio evolutivo e a natureza, arterial ou venosa. O tratamento das lesões menores e periféricas consiste, principalmente, na escleroterapia e excisão cirúrgica. Por outro lado, as lesões maiores e/ou intraósseas, requerem prévia embolização seguida da remoção cirúrgica. Há relatos do uso de fármacos, como o propranolol e corticoides sistêmicos, além da laserterapia, crioterapia e eletrocauterização. Hemangiomas na região de cabeça e pescoço são patologias complexas, relativamente comuns e a terapêutica deve ser direcionada individualmente, mediante as peculiaridades de cada caso.

Palavras-chave: Hemangioma; Neoplasias de tecido vascular; Escleroterapia.

\begin{abstract}
This study aimed to identify the main forms of treatment for head and neck hemangiomas. This is an integrative literature review, whose guiding question was the therapeutic approach to hemangiomas. A bibliographic survey was carried out in the Scielo and Pubmed databases, between the years 2009 to 2019. The search resulted in the selection of 28 articles, after which the following eligibility criteria were investigated: open access, publications in Portuguese, English or Spanish and studies whose titles and abstracts were in line with the purpose of this review. At the end, 5 articles were selected. Gray literature and duplicates were excluded. Upon critical analysis of the studies, it was
\end{abstract}


estimated that only 10 to $20 \%$ of hemangiomas require treatment. Therapeutic options for hemangioma are diverse, varying according to the characteristics of the patients and the peculiarities of the lesion, such as location, size, evolutionary stage and nature, arterial or venous. The treatment of minor and peripheral lesions consists mainly of sclerotherapy and surgical excision. On the other hand, larger and/or intraosseous lesions require previous embolization followed by surgical removal. There are reports of the use of drugs, such as propranolol and systemic corticosteroids, in addition to laser therapy, cryotherapy and electrocautery. Hemangiomas in the head and neck region are complex, relatively common pathologies and the therapy must be directed individually, according to the peculiarities of each case.

Keywords: Hemangioma; Vascular tissue neoplasms; Sclerotherapy.

\section{Resumen}

Este estudio tiene como objetivo identificar las principales formas de tratamiento de los hemangiomas de cabeza y cuello. Se trata de una revisión integradora de la literatura, cuya pregunta orientadora fue el abordaje terapéutico de los hemangiomas. Se realizó un relevamiento bibliográfico en las bases de datos Scielo y Pubmed, entre los años 2009 a 2019. La búsqueda resultó en la selección de 28 artículos, luego de lo cual se investigaron los siguientes criterios de elegibilidad: acceso abierto, publicaciones en portugués, inglés o español y estudios cuyos títulos y resúmenes concuerden con el propósito de esta revisión. Al final, se seleccionaron 5 artículos. Se excluyó la literatura gris y los duplicados. Tras un análisis crítico de los estudios, se estimó que solo del 10 al 20\% de los hemangiomas requieren tratamiento. Las opciones terapéuticas para el hemangioma son diversas, variando según las características de los pacientes y las peculiaridades de la lesión, como ubicación, tamaño, estadio evolutivo y naturaleza, arterial o venosa. El tratamiento de lesiones menores y periféricas consiste principalmente en escleroterapia y escisión quirúrgica. Por otro lado, las lesiones más grandes y / o intraóseas requieren una embolización previa seguida de una extirpación quirúrgica. Existen reportes del uso de fármacos, como propranolol y corticosteroides sistémicos, además de la terapia con láser, crioterapia y electrocauterio. Los hemangiomas en la región de cabeza y cuello son patologías complejas, relativamente frecuentes y el tratamiento debe ser dirigido individualmente, según las peculiaridades de cada caso.

Palabras clave: Hemangioma; Neoplasias de tejido vascular; Escleroterapia.

\section{Introdução}

O hemangioma infantil é uma neoplasia vascular benigna, caracterizada por proliferação celular aumentada ou malformação vascular, que está etiologicamente ligada a questões genéticas, prematuridade, idade materna avançada e traumas no local da lesão (DeHart \& Richter, 2019; Satterfield \& Chambers, 2019; Tiemann \& Hein, 2020). Em relação a localização anatômica, hemangiomas costumam ser encontrados na região de cabeça e pescoço, subglote, trato gastrointestinal, coluna vertebral, e no caso de acometimento oral, as áreas mais afetadas são língua, lábios e mucosa jugal (Cardoso et al., 2010; Araújo Filho et al., 2013).

Considerado o tumor mais comum da infância, o hemangioma, usualmente, tem uma fase de crescimento rápida e intensa proliferação celular, seguida por um período de involução (Azma \& Razaghi, 2018; DeHart \& Richter, 2019). Desse modo, 90\% dos casos sofrem remissão antes dos 9 anos de idade, ocorrendo em até 3\% das crianças (Queiroz et al., 2014) e 510\% dos casos surgem em crianças com até um ano de idade (Satterfield \& Chambers, 2019; Tiemann \& Hein, 2020). Porém, alguns casos podem regredir apenas na fase adulta (Azma \& Razaghi, 2018).

Apesar de, habitualmente, não provocar sintomatologia dolorosa, o hemangioma pode ocasionar úlceras, hemorragia, infecção secundária, dores pela compressão tecidual e deformação dos tecidos, dessa maneira, sendo necessária intervenção (Cardoso et al., 2010; Satterfield \& Chambers, 2019; Wang et al., 2019). Classificam-se de acordo com a profundidade, em superficiais, profundos ou mistos e baseada em sua localização, em focais ou multifocais (DeHart \& Richter, 2019). A associação com distúrbios funcionais ou de desenvolvimento, a classificação, extensão, localização e sintomatologia, além de características ligadas ao paciente, configuram a necessidade ou não de tratamento, que deve ter caráter individualizado (Cardoso et al., 2010; Satterfield \& Chambers, 2019). As terapêuticas tradicionais, escleroterapia e excisão cirúrgica, por vezes ocasionam complicações, desta forma, a laserterapia e outros tratamentos vem sendo utilizados (Azma \& Razaghi, 2018).

Mediante o exposto, é notória a importância do estudo da terapêutica do hemangioma, pelas variações apresentadas de acordo com as características do paciente, da lesão e comprometimento funcional ou de desenvolvimento. Desta forma, este 
estudo tem por finalidade identificar as principais formas de tratamento dos hemangiomas de cabeça e pescoço.

\section{Metodologia}

Trata-se de uma revisão integrativa da literatura, que sintetiza resultados de uma pesquisa desenvolvida com base em critérios de elegibilidade, posterior seleção das publicações e síntese, seguida de análise crítica e descritiva das informações encontradas (Pereira et al., 2018). A questão norteadora dessa pesquisa foi direcionada às principais abordagens terapêuticas relacionadas aos hemangiomas de cabeça e pescoço.

A coleta de dados foi realizada por meio de busca online, nas seguintes bases de dados: Scielo (Scientific Electronic Library Online) e PubMed (National Library of Medicine). Para conduzir essa seleção utilizaram-se os seguintes Descritores em Ciências da Saúde (Decs): Hemangioma; Neoplasias de tecido vascular; Escleroterapia, combinados aos operadores booleanos AND e OR.

Os critérios de inclusão utilizados foram: artigos científicos de acesso livre; disponíveis na íntegra em meio eletrônico; publicados entre 2009 e 2019; nos idiomas português, inglês ou espanhol; e, estudos cujos títulos e resumos mostraram-se em consonância com o propósito da revisão de literatura. Foram excluídas, a literatura cinzenta e duplicatas dos artigos. Ao final, selecionaram-se 5 artigos que atenderam aos critérios estabelecidos, destes 2 foram excluídos (Figura 1).

Figura 1. Fluxograma das etapas desenvolvidas para seleção dos estudos incluídos na revisão.
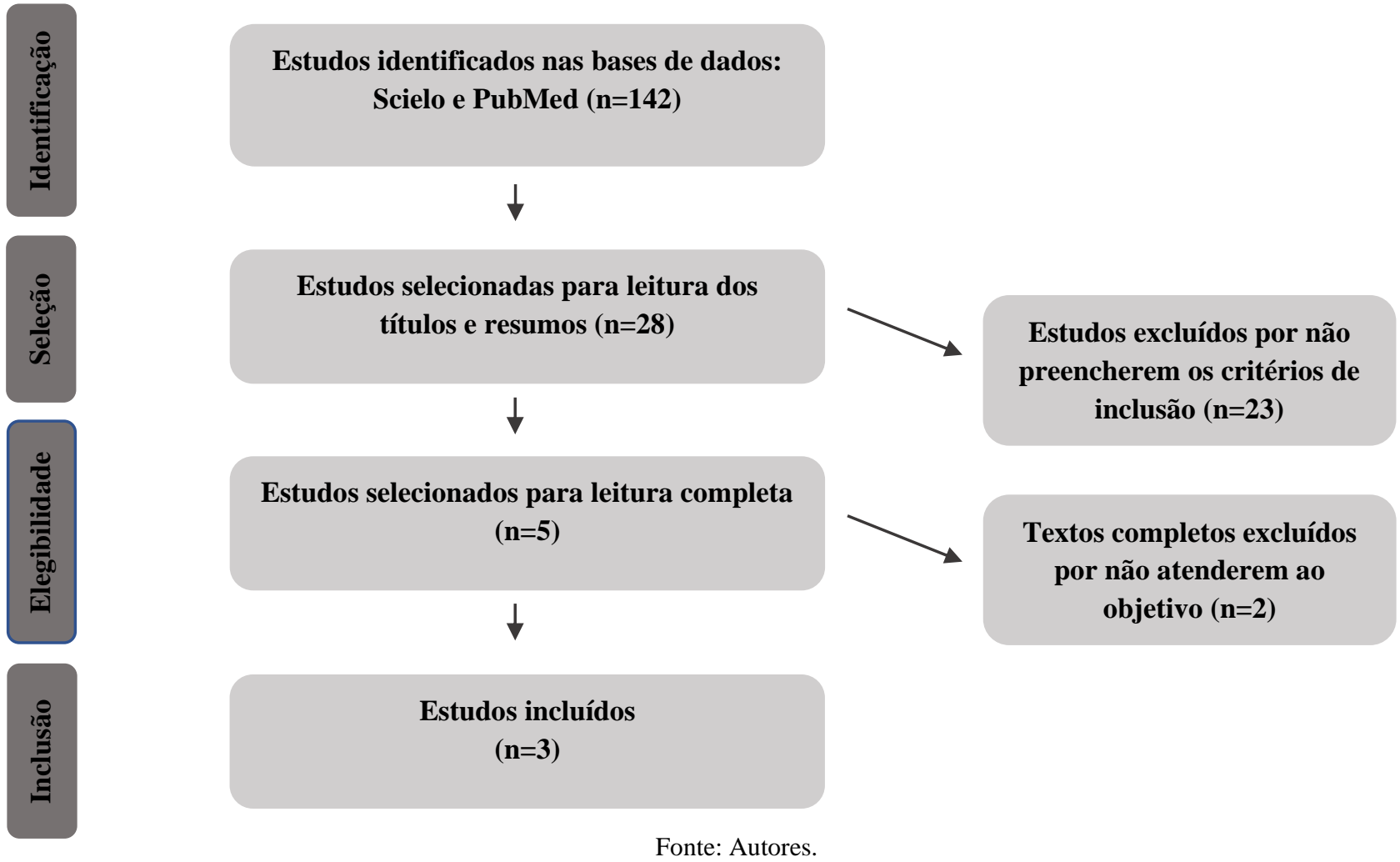

\section{Resultados}

Após um breve estudo bibliográfico, foram verificados 28 artigos nas bases de dados SCIELO e PUBMED, publicados entre os anos de 2009 a 2019. Destes, 23 foram excluídos por não estarem de acordo com os critérios de elegibilidade do presente estudo, restando apenas 5 artigos. Entres os 5 manuscritos elencados, 3 eram relatos de casos, sendo publicados respectivamente nos anos 2010, 2014 e 2019, um no idioma inglês e os demais em português (Tabela 1). 
Tabela 1. Descrição dos estudos selecionados quanto aos autores, ano de publicação, classificação da lesão e terapêutica.

\begin{tabular}{cccc}
\hline Autores & Ano & Classificação da lesão & Conduta terapêutica \\
\hline Cardoso et al. & 2010 & Hemangioma periférico & Excisão cirúrgica convencional \\
\hline Queiroz et al. & 2014 & Hemangioma periférico & $\begin{array}{c}\text { Escleroterapia (oleato de } \\
\text { monoetanolamina) }\end{array}$ \\
\hline Sahin, Sonmez, Yilmazbayhan \& Orhan & 2019 & Hemangioma cavernoso & $\begin{array}{c}\text { Remoção cirúrgica por abordagem } \\
\text { transnasal endoscópica }\end{array}$ \\
\hline
\end{tabular}

Fonte: Autores.

Os estudos selecionados em questão, mencionam três relatos de casos e as terapêuticas utilizadas para tratar o hemangioma. O tratamento do hemangioma varia conforme peculiaridades do paciente e da lesão, como o local do tumor, sua extensão e a presença ou ausência de sintomas (Pitta \& Gomes, 2009; Satterfield \& Chambers, 2019). Dessa maneira, é importante investigar cada caso isoladamente e analisar a procedência do tratamento a fim de evitar uma intervenção mais radical e contribuir para uma terapêutica conservadora.

Diante do que foi proposto no primeiro relato de caso, intitulado "Abordagem cirúrgica de hemangioma intraoral", que envolve uma lesão menor e periférica, optou-se por realizar uma excisão cirúrgica convencional, por meio do pinçamento e posterior remoção da lesão (Cardoso et al., 2010).

No segundo artigo selecionado, denominado "Tratamento de hemangioma oral com escleroterapia", o paciente relatou a presença da lesão há mais de dois anos, mencionou ainda o hábito de morder os lábios, e que, a partir disso, percebeu um aumento de tamanho da lesão, quando chorava ou nadava. Foi verificada uma lesão com coloração arroxeada, superfície lisa e mucosa íntegra. Como forma de tratamento optou-se pela escleroterapia, um método seguro que possibilita a regressão total ou parcial da lesão. Existem alguns agentes esclerosantes utilizados no método da escleroterapia, a exemplo de morruato de sódio, psilato de sódio, solução hipertônica de glicose e oleato de monoetanolamina. No caso citado, houve a aplicação do oleato de monoetanolamina, que promoveu a involução da lesão de modo rápido e seguro (Queiroz et al., 2014).

No terceiro manuscrito, nomeado "Hemangioma cavernoso em local incomum: fossa pterigopalatina", a paciente referiu cefaleia e incômodos na face. Após realização da tomografia computadorizada, observou-se uma lesão de tecido mole, bem circunscrita, que se estendia desde a fossa pterigopalatina esquerda até o seio cavernoso esquerdo. Em situações de emergência e/ou de insucesso aos tratamentos sistêmicos prévios, indica-se a cirurgia, por isso, nesse caso, optou-se pela remoção completa da lesão com o uso da abordagem transnasal endoscópica, sem emprego de embolização pré-operatória (Sahin et al., 2019).

\section{Discussão}

Hemangiomas são neoplasias vasculares benignas comuns na região da cabeça e do pescoço (Wang et al., 2019; Sahin et al., 2019). A maior incidência dessas neoplasias é relatada logo após o nascimento ou na primeira infância (Satterfield \& Chambers, 2019; Tiemann \& Hein, 2020). De acordo com alguns estudos, os hemangiomas apresentam proliferação endotelial, crescimento rápido e involução gradual, de modo que a maioria dos casos se resolve antes dos nove anos de idade (Azma \& Razaghi, 2018; DeHart \& Richter, 2019; Queiroz et al., 2014). A localização oral mais frequente dos hemangiomas é o lábio, mas outras áreas como língua, mucosa jugal e palato, também são referenciadas (Queiroz et al., 2014).

Ao exame físico, as lesões apresentam-se com coloração avermelhada ou arroxeada, de superfície lisa ou moriforme, e no geral, assintomáticas (Leung et al., 2021). Podem ser utilizadas manobras semiotécnicas, como a diascopia/vitropressão, para diferenciar as lesões vasculares das lesões pigmentadas. Durante a vitropressão, se ocorrer empalecimento da lesão e/ou diminuição do tamanho em razão do esvaziamento de seus vasos sanguíneos, há indícios de origem vascular, o que as 
diferencia de cistos e mucocele. Contudo, em áreas como gengiva e palato, a aplicação dessa técnica é dificultada (Cardoso et al., 2010; Queiroz et al., 2014).

A depender de sua localização e tamanho, além do prejuízo estético, essas neoplasias podem ocasionar assimetria facial, úlceras, hemorragia, infecção secundária, sintomatologia dolorosa, deformação dos tecidos e alterações funcionais (Atas et al., 2018; Satterfield \& Chambers, 2019; Wang et al., 2019). Para o diagnóstico de hemangiomas, a história clínica associada ao exame físico é fundamental, embora exames complementares também possam ser solicitados em alguns casos (Queiroz et al., 2014; DeHart \& Richter, 2019).

Nesse contexto, as lesões mais profundas podem ser analisadas através de exames de imagem, como tomografia computadorizada, ressonância magnética, radiografia e ultrassonagrafia com Doppler (Atas et al., 2018). A tomografia mostra a dimensão do tumor e/ou envolvimento ósseo, enquanto a ressonância revela a natureza vascular da lesão. Por sua vez, a ultrassonografia com Doppler demonstra a presença de irrigação arterial ou venosa na lesão. Assim, ao estudo Doppler, o hemangioma apresenta-se com uma discreta dilatação de vasos, baixo fluxo de resistência e natureza venosa (Queiroz et al., 2014).

No tocante ao tratamento dos hemangiomas, devem ser levados em consideração alguns fatores, como a idade do paciente, localização, tamanho, estágio evolutivo e a natureza, arterial ou venosa, da lesão (Pitta \& Gomes, 2009; Leung, Lam, Leong \& Hon, 2021). A maioria dos hemangiomas infantis não necessita ser tratado e, geralmente, esse tratamento se faz necessário quando há complicações (Leung et al., 2021). No geral, a terapêutica direcionada aos hemangiomas é classificada em duas categorias, local e sistêmica (Atas et al., 2018). Segundo Cardoso et al. (2010), os casos assintomáticos de hemangiomas devem ser acompanhados periodicamente.

De acordo com Queiroz et al., (2014), entre as opções terapêuticas para hemangiomas menores e periféricos estão a escleroterapia, excisão cirúrgica convencional, laserterapia, eletrocoagulação e crioterapia. Ainda segundo tais autores, lesões maiores e/ou intraósseas, localizadas em áreas estéticas, requerem prévia embolização ou obliteração da lesão e dos vasos adjacentes, para reduzir o tamanho da lesão antes da remoção cirúrgica completa (Queiroz et al., 2014).

Dentre os métodos terapêuticos anteriormente citados, a escleroterapia é uma opção segura, que possibilita a regressão total ou parcial da lesão, facilitando a ressecção cirúrgica posterior, caso ainda seja necessária (Queiroz et al., 2014). Já nos casos de pequenos hemangiomas intraorais, a crioterapia é considerada eficaz, com a vantagem de ser rápida e de gerar poucas complicações, quando realizada corretamente (Cardoso et al., 2010; Sakhiya et al., 2020).

Ademais, por muito tempo, o uso de corticoides sistêmicos foi considerado a principal terapêutica para os hemangiomas infantis, porém, tais medicações têm efeitos adversos insidiosos e de difícil controle, além de resposta variável (Bonini, Bellodi \& Souza, 2011). Nesse sentido, o interferon alfa tem sido utilizado com o intuito de limitar o crescimento da lesão, embora seu uso seja restrito pela neurotoxicidade gerada (Cardoso et al., 2010). Devido aos efeitos colaterais dos fármacos anteriormente citados, o propanol, outros $\beta$-bloqueadores e inibidores da enzima de conversão de angiotensina (ACE) vêm sendo amplamente utilizados no tratamento dos hemangiomas (Atas et al., 2018; Koh et al., 2020).

Com relação a terapia com propranolol, esta foi descoberta em 2008 e, desde então, é alvo de diversos ensaios clínicos, estudos coorte e revisões sistemáticas, que buscam fornecer informações sobre as indicações, dosagens, duração, eficácia e segurança da droga (Smithson et al., 2017; Yang et al., 2019; Koh et al., 2020). Todavia, efeitos adversos como possíveis danos ao cérebro (Moyakine et al., 2016) e a hipoglicemia (Yang et al., 2019), configuram preocupações e permanecem sem solução. Os índices de resolutividade do tratamento com propranolol variam de $82-100 \%$ (Marqueling et al., 2013), entretanto, existem efeitos adversos em torno de $17-96 \%$, com $3 \%$ desses efeitos exigindo suspensão imediata do propanolol (Koh et al., 2020).

Há relatos de outras terapias intervencionistas, como injeção de corticosteroides, laser e embolização, contudo, deve 
haver planejamento prévio conforme cada caso, pois existem contraindicações e riscos, como danos ao nervo óptico e aos músculos extraoculares (Bonini et al., 2011). Outra modalidade terapêutica é a eletrocauterização, considerada um método vantajoso pelo menor risco de hemorragia transoperatória, uma complicação temida na abordagem cirúrgica dos hemangiomas, e pela facilidade de execução da técnica (Cardoso et al., 2010).

De acordo com Pitta e Gomes (2009), a cirurgia é um dos pilares do tratamento dos hemangiomas e tem indicações específicas. Um dos riscos deste tratamento é a deformidade estética, que pode acarretar danos psicológicos aos pacientes. Outrossim, o procedimento cirúrgico pode acarretar danos nervosos, necrose tecidual, toxicidade sistêmica e hemorragias (Azma \& Razaghi, 2018). As indicações da cirurgia limitam-se a casos de emergência, de falha terapêutica ou por razões estéticas. A intervenção cirúrgica pode ser instituída através de embolização, ligação arterial seletiva ou exérese simples, com ou sem reconstrução plástica (Pitta \& Gomes, 2009).

Devido a complicações em terapias convencionais, a laserterapia tem sido introduzida para uso clínico no tratamento de lesões vasculares (Azma \& Razaghi, 2018; Sakhiya et al., 2020). Diferentes tipos de lasers são utilizados no tratamento de hemangiomas, os quais apresentam parâmetros distintos, no entanto, os mecanismos de atuação são semelhantes (Azma \& Razaghi, 2018; Jin et al., 2020). Dentre os mais utilizados, de forma isolada ou associada, destacam-se: o laser de dióxido de carbono (CO2), laser de argônio, laser de diodo e laser Erbium-YAG (Er: YAG) (Crisan et al., 2010; Sakhiya et al., 2020). Essa técnica apresenta algumas vantagens já que possibilita melhor controle do sangramento, promove uma cirurgia limpa e propicia um resultado estético superior (Sakhiya et al., 2020; Jin et al., 2020).

Diante disso, nota-se que os hemangiomas são lesões comuns de cabeça e pescoço e que se faz necessário conhecer seus aspectos clínicos, para que, assim, cada caso seja conduzido da melhor forma para o paciente (Cardoso et al., 2010).

\section{Conclusão}

Em suma, infere-se que as opções terapêuticas para o hemangioma são diversas, variando de acordo com características ligadas ao paciente e peculiaridades da lesão, como localização, tamanho, estágio evolutivo e a natureza, arterial ou venosa. No geral, a terapêutica pode ser local ou sistêmica. O tratamento das lesões menores e periféricas consiste, usualmente, na escleroterapia, excisão cirúrgica convencional, laserterapia, eletrocoagulação e crioterapia. Por outro lado, as lesões maiores e/ou intraósseas, requerem prévia embolização ou obliteração da lesão e dos vasos adjacentes, seguida da remoção cirúrgica. Nesse contexto, há também o tratamento sistêmico, baseado no uso de fármacos como corticoides, interferon alfa, propanol, outros $\beta$-bloqueadores e inibidores da enzima de conversão de angiotensina (ACE), todavia, alguns destes não são recomendados pelos efeitos adversos ocasionados.

Espera-se que essa revisão forneça subsídios para os cirurgiões-dentistas clínicos e acadêmicos de Odontologia sobre a terapêutica dos hemangiomas, além de esclarecer possíveis dúvidas, assim como conscientizar os mesmos sobre a importância do planejamento individualizado do tratamento dessa lesão. Recomenda-se que os novos estudos avaliem mais profundamente terapias contemporâneas, como a laserterapia, visando fornecer um maior embasamento aos clínicos e consequentemente, ampliar o leque de tratamento dos pacientes.

\section{Referências}

Araújo Filho, V. J. F., Cernea, C. R., \& Brandão, L. G. (2013). Manual do residente de cirurgia de cabeça e pescoço. (2a ed.), Manole.

Ataş, E., Koç, O., \& Artık, H. A. (2017). Clinical features and treatment results in children with hemangioma. Turk J Pediatr, 59 (3), 254-260. https://doi.org/10.24953/turkjped.2017.03.004

Azma, E., \& Razaghi, M. (2018). Laser Treatment of Oral and Maxillofacial Hemangioma. J Lasers Med Sci, 9 (4), 228-232. https://doi.org/10.15171/jlms.2018.41 
Bonini, F. K., Bellodi, F. S., \& Souza, E. M. (2011). Hemangioma infantil tratado com propanolol. An Bras Dermatol, 86 (04), 763-766. https://doi.org/10.1590/S0365-05962011000400022

Cardoso, C. L., Fernandes, L. M. P. S. R., Rocha, J. F., Gonçalves, E. S., Júnior, O. F., \& Taveira, L. A. A. (2010). Abordagem cirúrgica de hemangioma intraoral. Odontol Clin Cient, 9 (02), 177-180.

Crisan, B. V., Baciut, M., Baciut, G., Campian, R. S., \& Crisan, L. (2010). Laser treatment in oral and maxillofacial hemangioma and vascular malformations. TMJ. 60 (1), 34-38.

DeHart, A., \& Richter, G. (2019). Hemangioma: Recent Advances. F1000Res, 18 (08), 01-06. https://doi.org/10.12688/f1000research.20152.1

Jin, W. W., Tong, Y., Wu, J-M., Quan, H-H., \& Gao, Y. (2020). Observation on the effects of 595- nm pulsed dye laser and 755- nm long-pulsed alexandrite laser on sequential therapy of infantile hemangioma. J Cosmet Laser Ther, 22 (3), 159-164. https://doi.org/10.1080/14764172.2020.1783452

Koh, S. P., Leadbitter, P., Smithers, F., \& Tan, S. T. (2020). 3 -blocker therapy for infantile hemangioma. Expert Rev Clin Pharmacol, 13 (8), 899-915. https://doi.org/10.1080/17512433.2020.1788938

Leung, A. K. C., Lam, J. M., Leong, K. F., \& Hon, K. L. (2021). Infantile hemangioma: an updated review. Curr Pediatr Rev, 17 (1), 55-69. https://doi.org/ $10.2174 / 1573396316666200508100038$.

Marqueling, A. L., Oza, V., Frieden, I. J., \& Puttgen, K. B. (2013). Propranolol and infantile hemangiomas four years later: a systematic review. Pediatr Dermatol, 30 (2), 182-191. https://doi.org/10.1111/pde.12089

Moyakine, A. V., Kerstjens, J. M., Koulil, S. S-v. \& Vleuten, C. J. M. (2016). Propranolol treatment of infantile hemangioma (IH) is not associated with developmental risk or growth impairment at age 4 years. J Am Acad Dermatol, 75 (1), 59-63. https://doi.org/10.1016/j.jaad.2016.02.1218

Pereira, A. S., Shitsuka, D. M., Parreira, F. J. \& Shitsuka, R. (2018). Metodologia da Pesquisa Científica. UFSM.

Pitta, G. B. B., \& Gomes, R. R. (2009). Tratamento de hemangioma ulcerado: relato de caso. J Vasc Bras, 8 (03), 263-266. https://doi.org/10.1590/S167754492009005000010

Queiroz, S. I. M. L., Assis, G. M., Silvestre, V. D., Germano, A. R., \& Silva, J. S. P. (2014). Tratamento de hemangioma oral com escleroterapia: relato de caso. J Vasc Bras, 13 (03), 249-253. https://doi.org/10.1590/jvb.2014.035

Sahin, B., Sonmez, S., Yilmazbayhan, E. D., \& Orhan, K. S. (2019). Cavernous hemangioma in unusual location: pterygopalatine fossa. Braz J Otorhinolaryngol, 85 (01), 121- 124. https://doi.org/10.1016/j.bjorl.2016.02.003

Sakhiya, J., Sakhiya J, D., Virmani, N., Patel, A., Daruwala, F., \& Dudhatra, N. A challenging case of cavernous hemangioma of the face treated with the combination of cryotherapy, laser, and dermatosurgery. J Clin Aesthet Dermatol, 13 (8), 14-16.

Satterfield, K. R., \& Chambers, C. B. (2019). Current treatment and management of infantile hemangiomas. Surv Ophthalmol, 64 (5), 608-618. https://doi.org/ 10.1016/j.survophthal.2019.02.005

Smithson, S. L., Rademaker, M., Adams, S., Bade, S., Bekhor, P., Davidson, S., \& Phillips, R. (2017). Consensus statement for the treatment of infantile haemangiomas with propranolol. Australas J Dermatol, 58 (2), 155-159. https://doi.org/10.1111/ajd.12600

Tiemann, L., \& Hein, S. (2020). Infantile Hemangioma: A review of current pharmacotherapy treatment and practice pearls. J Pediatr Pharmacol Ther, 25 (7), 586-599. https://doi.org/10.5863/1551-6776-25.7.586.

Wang, Q., Xiang, B., Chen, S., \& Ji, Y. (2019). Efficacy and safety of oral atenolol for the treatment of infantile haemangioma: A systematic review. Australas J Dermatol, 60 (3), 181-185. https://doi.org/10.1111/ajd.12966

Yang, H., Hu, D-L., Shu, Q., \& Guo, X-D. (2019). Efficacy and adverse effects of oral propranolol in infantile hemangioma: a meta-analysis of comparative studies. World J Pediatr, 15 (6), 546-558. https://doi.org/10.1007/s12519-019-00285-9 